\title{
Identification of objectionable flavors in purported spontaneous oxidized flavor bovine milk
}

\author{
David M. Potts* and Devin G. Peterson ${ }^{1}$ \\ ${ }^{*}$ Department of Food Science and Nutrition, University of Minnesota, St. Paul 55108 \\ †Department of Food Science and Technology, The Ohio State University, Columbus 43210
}

\begin{abstract}
Spontaneous oxidized flavor (SOF) has been reported over the past 5 decades as a sporadic objectionable flavor problem in bovine milk. Parameters previously reported to influence SOF development in milk have been contradictory, limiting the ability to monitor and develop mitigation strategies. The current paper investigates the causative compounds associated with milk identified as SOF milk in the Midwest dairy region of the United States. Based on GC/MS-olfactometry analysis, endo-borneol, 2-methylisoborneol, and $\alpha$-terpineol were identified as the off-flavor compounds. Sensory recombination studies further confirmed the sensory contribution of these compounds to the noted off-flavor attributes in the original milk, which were described as "green," "musty," and "unclean." These compounds are known microbial-derived flavor taints, indicating oxidation was not the origin of the objectionable flavor in the milk. This noted misclassification of the milk as SOF indicates the challenge of defining flavor defects without the identification of the active compounds.
\end{abstract}

Key words: milk, off-flavor, oxidation, spontaneous oxidized flavor, microbial taint

\section{INTRODUCTION}

Spontaneous oxidized flavor (SOF) in bovine milk has been identified in recent decades as a sporadic off-flavor problem affecting milk both before and after pasteurization. It is primarily characterized by "cardboard," "fishy," or "oxidized" flavors, and it is often suggested as capable of contaminating the flavor of otherwise acceptable milk during bulk tank mixing before pasteurization. The off-flavor was first identified in northern Europe, and during the last decade it has been recognized as a growing problem in the Midwest-

Received May 11, 2018.

Accepted August 1, 2018.

${ }^{1}$ Corresponding author: peterson892@osu.edu ern United States. It has been frequently described as a seasonal off-flavor taint, appearing in the late autumn months and disappearing in early spring (Juhlin et al., 2010a). Because the associated objectionable flavors are reminiscent of lipid oxidation and affect the flavor of clean milk, a common assumption is an oxidative radical mechanism is responsible, hence the name SOF. Researchers have frequently attempted to correlate SOF sensory defects with changes in known causative factors associated with oxidative reaction pathways. The most common examples include changes in bovine diet, such as the presence of specific PUFA, as well as other factors that affect instigation or inhibition of SOF through various oxidizers, antioxidants, catalysts, and so on (Dunkley et al., 1963; Bruhn et al., 1976; Juhlin et al., 2010a).

Several studies have suggested that the presence of particular micronutrients such as copper may catalyze lipid oxidation products in SOF milk. Juhlin et al. (2010a) claimed that the problem might be partially genetic, especially in the variability of copper levels during lactation of individual cows. In a later study, the group also asserted that a significant correlation was present between PUFA, copper levels, and SOF formation, although they noted that precisely defining the levels of substrates and pro- and antioxidants directly responsible was exceedingly complex and required further study (Juhlin et al., 2010b). Timmons et al. (2001) further described a link between increased SOF with higher levels of PUFA combined with a higher presence of copper within milk.

Others have attributed SOF formation exclusively to lipid-antioxidant levels and interactions. Clausen et al. (2010) found that higher concentrations of initial lipid peroxidation products, in combination with reduced levels of lower molecular weight antioxidants in the initiation stages of lipid oxidation, could accelerate oxidized flavor defects. In contrast, Granelli et al. (1998) investigated levels of PUFA, tocopherol, and $\beta$-carotene levels, concluding that their control herds showed lower antioxidant:PUFA ratios than experimental herds, yet did not develop any characteristic SOF flavor. 
A limitation of prior studies focused on SOF instigation and inhibition has been the lack of research focused on the identification of active compounds. Knowledge of the compounds responsible for the flavor defects in SOF milk would lend further insight regarding the potential source and enabling the development of effective mitigation strategies. Furthermore, it also provides a basis to analytically monitor for product defects. The overall objective of this study was to identify the compounds responsible for the flavor defects in the milk sample obtained from the Midwestern US region flagged as SOF by Midwest Dairy Foods Research Center.

\section{MATERIALS AND METHODS}

\section{Milk Samples}

Nonflavor defective (control) and off-flavored milk samples labeled as SOF were obtained through the Midwest Dairy Foods Research Center (St. Paul, MN). The SOF labeled milk was obtained from a farm in rural Nebraska flagged by producers for several consecutive years as having a noticeable, cardboard/oxidized SOF-type flavor taint arising from Jersey and Holstein cows between late autumn and early spring. Both the control and SOF labeled raw whole milk samples were shipped in chilled containers to the University of Minnesota for analysis. Upon arrival, samples were evaluated (and expectorated) by 3 experienced trained dairy judges at the University of Minnesota (Department of Food Science and Nutrition) to evaluate overall flavor quality. The control milk was noted as having typical flavors associated with high-quality whole fat milk, and the SOF labeled milk was noted as having "unclean" or poor flavor quality and aftertaste, as well as a metallic sensation. Milk samples were HTST pasteurized at the University of Minnesota Joseph Warthesen Food Processing Center (St. Paul, MN) using a HTST/UHT Direct Steam Injection \& Indirect Tubular Processing System (MicroThermics, Raleigh, NC). Processing conditions included sanitation using a chlorine solution $(100 \mathrm{ppm})$ followed by a clean water wash at $248^{\circ} \mathrm{F}$ $\left(120^{\circ} \mathrm{C}\right)$ for $1 \mathrm{~h}$. Control and SOF labeled milk samples were individually homogenized and processed to a final temperature of $165^{\circ} \mathrm{F}\left(74^{\circ} \mathrm{C}\right)$ for $20 \mathrm{~s}$ to ensure proper pasteurization. Previously sterilized glass bottles were filled with respective samples and cooled immediately to $60^{\circ} \mathrm{F}\left(16^{\circ} \mathrm{C}\right)$. Next, samples were tasted by 3 experienced dairy judges to evaluate flavor quality of the control and SOF labeled samples postpasteurization. The judges identified the control milk as "clean," or containing no objectionable volatile notes; the SOF labeled sample, however, was identified as retaining the "unclean" flavor profile with a metallic and objection- able aftertaste. The pasteurized samples were either immediately prepared for analysis (d 0 samples) or placed under refrigerated storage $\left(4-5^{\circ} \mathrm{C}\right)$ for 2 wk before subsequent extraction and analysis (d 14 samples). A standard plate count methodology was conducted during the 2-wk period using a Plate Count Agar (Neogen Corporation, Lansing, MI) to observe overall colony-forming units per milliliter and ensure adequate pasteurization of samples. For both control and SOF labeled milks, plate counts were initially calculated as too few to count and $2,400 \mathrm{cfu} / \mathrm{mL}$, respectively; both fell within the $<20,000 \mathrm{cfu} / \mathrm{mL}$ limits for grade A pasteurized milk as dictated by the United States Pasteurized Milk Ordinance. After $14 \mathrm{~d}$ of storage, plate counts were calculated as 1,800 and 49,000 cfu/mL for control and off-flavored milks, respectively.

\section{Chemicals}

Authentic standards used for quantification in this study included E-2-decenal, 2-heptanone, nonanal, 2-nonanone, 2-methylisoborneol, 2-methyl-3-heptanone, $\alpha$-terpineol, E,E-2,4-decadienal, 2-methylvaleric acid, and endoborneol (Sigma-Aldrich, St. Louis, MO). Diethyl ether was obtained from Fisher Scientific (Somerville, NJ).

\section{Extraction Procedure}

A modified version of the methodologies reported by Karagül-Yüceer et al. (2001) and Colahan-Sederstrom and Peterson (2005) were incorporated for the volatile extraction of control and SOF labeled milks. Samples were extracted in duplicate after pasteurization, both fresh (d 0) and after 2 wk (d 14) to observe storage effects on any off-flavors present, particularly because SOF has historically been reported to exacerbate over time. One liter of redistilled diethyl ether was spiked with internal standard solutions: $3 \mu \mathrm{L}$ of a 2-methyl3-heptanone internal standard solution $(50 \mu \mathrm{L} / 5 \mathrm{~mL}$ of diethyl ether) for the neutral/basic volatile extract, and $3 \mu \mathrm{L}$ of a 2 -methylvaleric acid internal standard solution $(33 \mu \mathrm{L} / 5 \mathrm{~mL}$ of diethyl ether) for the acidic volatile extract. One liter of each respective milk sample was measured and divided into 5 individual Teflonlined centrifuge bottles with Tefzel closures (Nalgene, Rochester, NY). Twenty grams of sodium chloride was added in each bottle, followed by addition of $40 \mathrm{~mL}$ of the redistilled diethyl ether with the internal standards (Fisher Scientific, Fair Lawn, NJ). The milk in each bottle was individually extracted 5 times $(40 \mathrm{~mL} /$ bottle, $5 \times 200 \mathrm{~mL}$ extractions). After solvent addition, each bottle was gently overturned several times, 
followed by mild stirring on an orbital shaking table (model 3540, Labline Instruments Inc., Melrose Park, IL) at 30\% maximum speed for $20 \mathrm{~min}$, and gently overturned several times again to avoid emulsion formation. The samples were centrifuged for $20 \mathrm{~min}$ at $4^{\circ} \mathrm{C}$ at $3,000 \times g$ (Allegra X-22R Centrifuge, Beckman Coulter, Brea, CA). After centrifugation, the solvent was carefully removed and pooled, dried over anhydrous sodium sulfate, filtered, and stored overnight in a $-20^{\circ} \mathrm{C}$ freezer. Samples were then concentrated to approximately $100 \mathrm{~mL}$ using a fractional distillation column. The volatile fraction was then isolated using a solvent-assisted flavor evaporation system as described by Engel et al. (1999). The resulting volatile fractions were further concentrated to approximately $20 \mathrm{~mL}$, after which they were additionally fractionated into neutral/basic and acidic fractions. The ether samples were washed with $1 M$ sodium bicarbonate $(3 \times 50$ $\mathrm{mL}$ ) and a saturated solution of sodium chloride in water $(2 \times 55 \mathrm{~mL})$ using a separatory funnel. The organic phase containing the neutral/basic volatiles was isolated and dried over anhydrous sodium sulfate and further distilled to a volume of approximately $1 \mathrm{~mL}$. The aqueous fractions were combined and reacidified to a $\mathrm{pH}$ of 1.5 with $18 \% \mathrm{vol} / \mathrm{vol}$ of $12 M$ hydrochloric acid and extracted with repurified diethyl ether $(3 \times 50$ $\mathrm{mL})$ to isolate the acidic volatile fraction. The fraction was dried over anhydrous sodium sulfate and distilled to approximately $1 \mathrm{~mL}$. Extracts were stored at $-80^{\circ} \mathrm{C}$ before analysis.

\section{Gas Chromatography-Mass Spectrometry}

Flavor extracts were analyzed with an Agilent 7890A GC (Agilent Technologies, Santa Clara, CA) equipped with a Leco Pegasus 4D GCXGC 4D TOF-MS system, as well as a Agilent Technologies 6890 GC equipped with a Agilent Technologies 5973 MSD. Two $\mu \mathrm{L}$ of each sample was injected under splitless mode; inlet temperature was set at $250^{\circ} \mathrm{C}$, and helium was used as a carrier gas with total flow rate of $1.0 \mathrm{~mL} / \mathrm{min}$. Compounds were analyzed on 2 different GC capillary columns with different chemistries: a DB-WAX (Agilent, $60 \mathrm{~m} \times 0.25 \mathrm{~mm} \times 0.25 \mu \mathrm{m}$ ) and HP-5 (Agilent, $60 \mathrm{~m} \times 0.25 \mathrm{~mm} \times 0.25 \mu \mathrm{m})$. The temperature profile used for the DB-WAX column was $40^{\circ} \mathrm{C}$ for $2 \mathrm{~min}$, then gradually increased to $220^{\circ} \mathrm{C}$ at $3^{\circ} \mathrm{C} / \mathrm{min}$ and was held for $3 \mathrm{~min}$; for the HP-5 the oven temperature profile started at $40^{\circ} \mathrm{C}$ and was held for $2 \mathrm{~min}$, then gradually increased to $250^{\circ} \mathrm{C}$ at $3^{\circ} \mathrm{C} / \mathrm{min}$ and was held for $3 \mathrm{~min}$. Mass spectral data were obtained in scan mode with a range of 30 to 350 amu. Confirmation of compounds was performed using authentic standards coupled with mass spectral data.

\section{Gas Chromatography-Mass Spectrometry/Olfactometry}

Flavor extracts were analyzed using an Agilent Technologies 6890A GC coupled with an Agilent Technologies $5973 \mathrm{MS}$ detector and equipped with an olfactometry port (Gerstel, Baltimore, MD). Chromatographic separation was performed on a DB-WAX or an HP-5 column $(60 \mathrm{~m} \times 0.25 \mathrm{~mm} \times 0.25 \mu \mathrm{m})$. One microliter of each sample was injected under splitless mode with the inlet temperature set at $250^{\circ} \mathrm{C}$. The temperature profile used for the DB-WAX column was $40^{\circ} \mathrm{C}$ for 2 min, then gradually increased to $230^{\circ} \mathrm{C}$ at $5^{\circ} \mathrm{C} / \mathrm{min}$ and held for 3 min. For the HP-5 column, the temperature profile was $40^{\circ} \mathrm{C}$ for $2 \mathrm{~min}$, then gradually increased to $250^{\circ} \mathrm{C}$ at $3^{\circ} \mathrm{C} / \mathrm{min}$ and held for $3 \mathrm{~min}$. After separation, the effluent of each column was split 1:1 between an MS and olfactory sniffing port. Purified air was bubbled through distilled water and purged at the end of the sniffing port at a rate of $10 \mathrm{~mL} / \mathrm{min}$. Three panelists experienced in GC-MS/olfactometry analysis protocols identified aromas as they eluted from the heated olfactory sniffing $\operatorname{arm}\left(250^{\circ} \mathrm{C}\right)$. During olfactory runs, panelists recorded the elution time of the compound, perceived odor descriptor, and intensity on a scale from 1 to 5, based on the OSME method for GC-olfactometry (McDaniel et al., 1990). Intensity ratings were assigned numerical values as follows: $1=$ very weak; $2=$ weak; 3 $=$ moderate; $4=$ strong; and $5=$ very strong.

Data from each panelist were compiled and values were averaged among all 3 individuals. Compounds were positively identified using mass spectral data, odor descriptors, and Kovats retention indices in comparison with authentic standards. Compounds were labeled as "tentatively identified" if mass spectra were not obtained.

\section{Quantitation of Selected Off-Flavor Compounds}

The selected compounds endo-borneol, 2-methylisoborneol, $\alpha$-terpineol, 1-nonanal, 2-heptanone, 2-nonanone, E-2-decenal, and E,E-2,4-decadienal were quantified in the SOF labeled and control milk samples. Analyses were performed on an Agilent Technologies 6890A GC coupled with an Agilent Technologies 5973 MS detector using either scan mode (30-350 amu) or selective ion monitoring mode for maximum sensitivity on a HP-5 column. One microliter of each sample was injected under splitless mode with the inlet temperature set at $250^{\circ} \mathrm{C}$. The temperature profile used was $40^{\circ} \mathrm{C}$ for 2 min, then gradually increased to $250^{\circ} \mathrm{C}$ at $7^{\circ} \mathrm{C} / \mathrm{min}$ and held for $3 \mathrm{~min}$. Helium was used as a carrier gas and set at constant flow at $1.0 \mathrm{~mL} / \mathrm{min}$. A combination of internal and external standard protocols was used. 
Quantitative analysis was completed using comparison of peak areas and the calculation of retention factors using the internal standards described above. Percent recovery studies were performed in triplicate and calculated for each of the individual compounds of interest, and the final calculated odorant concentrations were comparable to the concentrations obtained from independent 5-point calibration curves for each compound $\left(\mathrm{R}^{2}>0.97\right.$ and good linearity observed for all compounds). For selective ion monitoring analyses, ions characteristic to the odorants of interest were selected and are reported in Table 1. Statistical significance was verified using the Student's t-test. All analyses were performed with Statistix 10 (Analytical Software, Tallahassee, FL). Statistical significance was established at $P<0.05$ for all compounds.

\section{Sensory Recombination Study}

Approval for the sensory evaluation protocol was granted by the Ethics Committee, University of Minnesota (IRB \#1510E79045). An 11-member sensory panel was convened, consisting of individuals familiar with sensory evaluation protocols for various food products. Panelists participated in a degree of difference sensory test. They were provided with $30 \mathrm{~mL}$ of commercially available whole milk as a reference. Panelists were served milk samples randomly assigned with 3-digit codes. For each test, panelists were presented with 3 different milk samples: control whole milk sample (same as the reference milk) and 2 recombinant SOF labeled milk model samples (control milk with added compounds) that consisted of the objectionable flavors (identified by GC-MS/olfactometry analysis) at concentrations at either d 0 or 14 . The control milk was defined as a high quality, commercially available whole bovine milk. Panelists were asked to taste the reference milk, followed by each of the 3 randomly coded samples from left to right and evaluate them for degree of overall flavor difference from the reference (not appearance or texture). Panelists were instructed to rate the milks on a 5 -point scale, ranging from $0=$ no difference, $1=$ very slight difference, $2=$ slight difference, $3=$ moderate difference, $4=$ large difference, or $5=$ extremely large difference. Panelists were additionally asked to describe the perceived flavor differences noticed in the samples. Data were analyzed using a 2-sided Dunnett's multiple comparisons test, using the statistical software package Statistix 10 (Analytical Software).

\section{RESULTS AND DISCUSSION}

An objectionable flavored milk sample was obtained from a farm in the Midwestern United States for
Table 1. Select aroma compounds quantified for concentration comparison in control and off-flavored milk samples ${ }^{1}$

\begin{tabular}{lcc}
\hline Compound name & $\begin{array}{c}\text { Molecular } \\
\text { weight }\end{array}$ & $\begin{array}{c}\text { Monitored ions } \\
\text { (SIM) }\end{array}$ \\
\hline 2-Methyl-3-heptanone $\left(\mathrm{I}_{\text {std }}\right)$ & 128 & 128 \\
Nonanal & 142 & 98,142 \\
Endo-borneol & 154 & 95,154 \\
2-Methylisoborneol & 168 & $95,107,168$ \\
--Terpineol & 154 & 93,154 \\
E-2-Decenal & 154 & 97,154 \\
E,E-2,4-Decadienal & 152 & 81,152 \\
\hline
\end{tabular}

${ }^{1}$ Corresponding values for average compound molecular weights and ions monitored during GC-MS selective ion monitoring (SIM)-mode analysis for respective peak areas.

analysis. The Midwest Dairy Foods Research Center designated the milk as SOF based on the documented seasonality (over 2 consecutive years) and noted flavor descriptors ("oxidized," "cardboard," "fishy," and so on; Shipe et al., 1978; Nicholson, 1993; Juhlin et al., 2010b). Thus, elevated levels of lipid oxidation species were expected.

The aroma profile of the pasteurized SOF milk and a control milk (no apparent defects) after 0 and 14 d of storage were characterized by GC-MS/olfactometry analysis. Many of the compounds identified were common to both samples and are typical of fresh, high quality milk, including fatty acids, aldehydes, ketones, and lactones (shown in Tables 2-5; Villeneuve et al., 2013). The acidic fraction volatile compounds identified in the SOF labeled milk and control samples at $\mathrm{d} 0$ and 14 are shown in Tables 2 and 3, respectively. The odor intensities and peak areas (data not shown) revealed only negligible differences in the profiles of the known hydrolytic lipid oxidation products between the samples and during storage. The acids identified were typical to milk and associated with "fatty" or "cheese-like" aroma descriptors. Therefore, the free fatty acid compounds were not considered as the source of the objectionable flavor and were not further evaluated.

The odor-active compounds detected in the neutral/ basic fraction of the SOF labeled and control milk samples at d 0 and 14 are shown in Tables 4 and 5, respectively. Typical in milk, lipid peroxidation compounds were identified with descriptors such as green and fatty, as well as hydrolytic thermally derived methyl ketone products. In general, many of the compounds identified in the SOF sample were also present in the control milk; however, several key differences were also observed. As a first part of the analysis, differences in the noted lipid oxidation products were investigated. Two criteria were established to select lipid oxidation products for quantification: (1) observed $>30 \%$ peak area (total ion current-MS) in SOF versus control or (2) observed an increase of $>30 \%$ in peak area (total 
Table 2. Aroma compounds identified in the acidic fraction of control milk ${ }^{1}$

\begin{tabular}{|c|c|c|c|c|c|}
\hline Identified compound & DB-WAX ${ }^{2}$ & $\mathrm{HP}-5^{2}$ & Odor descriptor & \multicolumn{2}{|c|}{ Average OSME intensity } \\
\hline Acetic acid & 1490 & 594 & Fat, milk, cheese & 2.3 & 2.2 \\
\hline Butyric acid & 1597 & 846 & Rancid oil & 3.2 & 2.2 \\
\hline Pentanoic acid & 1722 & 976 & Cheese & 2.3 & 2.2 \\
\hline Hexanoic acid & 1803 & 1022 & Soap, sweat & 2.3 & 2.7 \\
\hline Benzoic acid & 2434 & - & Fat, rancid & 1.8 & 2.0 \\
\hline Dodecanoic acid & 2483 & - & Fat & 1.8 & 2.5 \\
\hline
\end{tabular}

${ }^{1}$ Identified on 2 column chemistries with corresponding odor descriptors and OSME odor intensity values from d 0 and 14 post-HTST pasteurization, averaged among 3 panelists.

${ }^{2}$ DB-WAX and HP-5 columns were from Agilent Technologies, Santa Clara, California.

ion current-MS) over the 14-d period of storage (data not shown) in the SOF labeled sample. Accordingly, 5 compounds were selected and are shown in Figure 1. Nonanal and E,E-2,4-decadienal were only observed in the SOF milk, at concentrations of 9.1 to 11 and 0.41 to $0.91 \mu \mathrm{g} / \mathrm{L}$, respectively. These values were above their published odor threshold concentrations $(1 \mu \mathrm{g} / \mathrm{L}$ and 0.07-0.2 $\mu \mathrm{g} / \mathrm{L}$, respectively; Buttery et al., 1988; Plotto et al., 2004). However, decadienal decreased significantly over the 14-d storage period, whereas no change in concentration was noted for nonanal. The other 3 compounds, 2-heptanone, 2-nonanone, and E-2-decenal were not detected in the $\mathrm{d} 0$ samples but were present after $14 \mathrm{~d}$. The concentrations of both 2-heptanone and 2-nonanone (1.9 and $1.5 \mu \mathrm{g} / \mathrm{L}$, respectively) were below the lowest published literature odor threshold values in water $(140$ and $5 \mu \mathrm{g} / \mathrm{L}$, respectively; Teranishi et al., 1974). The concentrations of E-2-decenal $(0.69 \mu \mathrm{g} / \mathrm{L})$ was slightly above its published aqueous odor threshold of $0.3 \mu \mathrm{g} / \mathrm{L}$ (Buttery et al., 1988). However, considering whole milk contains 3 to $4 \%$ fat, the threshold values of detection would be expected to increase (higher concen- tration) for these lipophilic compounds in comparison to a water base. The lack of unique lipid oxidation compounds in the SOF labeled milk above the detection threshold, as well as no observed general increase in lipid oxidation compounds during storage, suggested the traditional SOF hypothesis was not contributing to the objectionable flavor defect.

Further review of the odor-active compounds detected in the neutral/basic fraction revealed the SOF labeled milk had a distinct series of terpenoid compounds with characteristic "green" and "musty" descriptors, that included endo-borneol, 2-methylisoborneol, and $\alpha$-terpineol (Table 5). These compounds were not detected in the control milk, suggesting possible involvement in the perceived objectionable flavor reported in the SOF labeled milk. These terpenoid compounds are known flavor taints that originate from bacterial and fungal sources. For example, 2-methylisoborneol has been reported to bioaccumulate in catfish, resulting in sensory defects that originated from cyanobacteria (blue-green algae) in aqueous environments (Schrader and Summerfelt, 2010). Siegmund and Pöllinger-Zierler

Table 3. Aroma compounds identified in the acidic fraction of spontaneous oxidized flavor labeled milk ${ }^{1}$

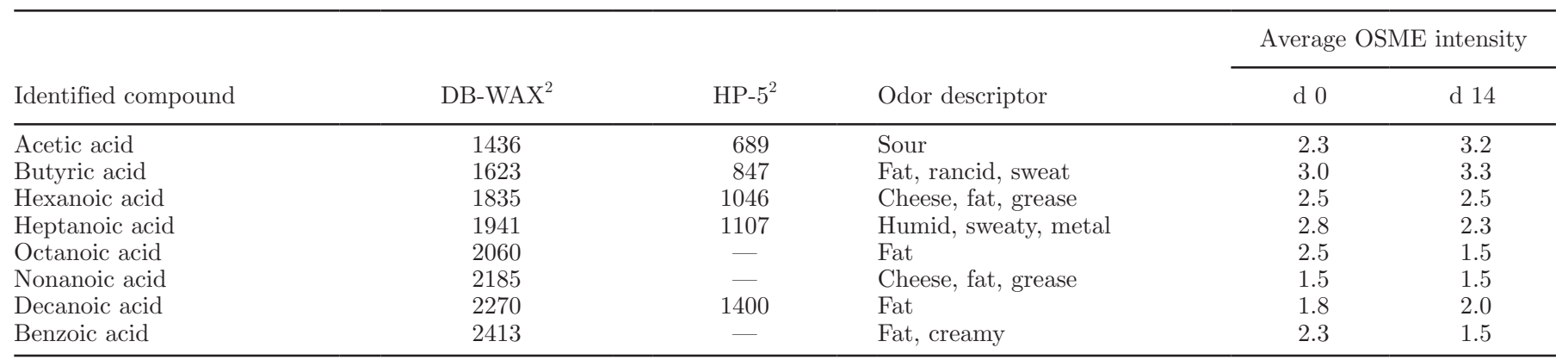

${ }^{1}$ Identified on 2 column chemistries with corresponding odor descriptors and OSME odor intensity values from d 0 and 14 post-HTST pasteurization, averaged among 3 panelists.

${ }^{2}$ DB-WAX and HP-5 columns were from Agilent Technologies, Santa Clara, California. 
Table 4. Aroma compounds identified in the neutral/basic fraction of control milk ${ }^{1}$

\begin{tabular}{|c|c|c|c|c|c|}
\hline Identified compound & DB-WAX ${ }^{2}$ & $\mathrm{HP}-5^{2}$ & Odor descriptor & \multicolumn{2}{|c|}{ Average OSME intensity } \\
\hline Acetic acid & 1450 & 607 & Fat, cheese, pungent & - & 2.8 \\
\hline Isobutyl acetate & - & 775 & Wheat, almond & 2.0 & 1.7 \\
\hline Hexanal & 1084 & 804 & Wheat, dough, grain & - & 2.2 \\
\hline 1-Nonen-3-one & 1239 & 836 & Fat, milkfat & 2.3 & 3.0 \\
\hline Pentylfuran & 1245 & 999 & Earthy, green, rubbery & 2.3 & 2.5 \\
\hline Benzyl alcohol & - & 1034 & Roasted, meaty & 2.3 & 1.3 \\
\hline 1-Octanol & 1547 & 1061 & Sulfury, pungent, barn & 2.2 & 1.5 \\
\hline 2-Nonanone & 1390 & 1091 & Milk, fat & 2.2 & 3.0 \\
\hline Nonanal & 1400 & 1106 & Milk, fat, dairy & 2.2 & 3.3 \\
\hline Menthol & 1646 & 1180 & Green & - & 2.3 \\
\hline Thymol & 2242 & 1293 & Herbal & 2.3 & 2.8 \\
\hline Geranyl acetone & - & 1444 & Green, musty & 2.5 & 2.8 \\
\hline$\delta$-Decalactone & 2235 & 1453 & Sweet, vanilla & 3.2 & 2.7 \\
\hline$\gamma$-Decalactone & 2135 & 1483 & Lactone, peach & 2.8 & 2.7 \\
\hline Methyl laurate & 1786 & 1511 & Fatty & 3.7 & 2.8 \\
\hline
\end{tabular}

${ }^{1}$ Identified on 2 column chemistries with corresponding odor descriptors and OSME odor intensity values from d 0 and 14 post-HTST pasteurization, averaged among 3 panelists.

${ }^{2}$ DB-WAX and HP-5 columns were from Agilent Technologies, Santa Clara, California.

(2006) identified a wide range of "musty" compounds present in apple juice both before and after pasteurization including endo-borneol, 2-methylisoborneol, and $\alpha$-terpineol. In this study, populations of various thermoacidophilic bacteria (Alicyclobacillus acidoterrestris, Actinomycetes, and Streptomyces spp.) were found to quantitatively increase over time on the surfaces of apples, which corresponded with increasing sensory defects in apple juice produced from the contaminated fruit. In general, terpenoid compounds have been noted in literature as having exceedingly low odor thresholds (endo-borneol at $140 \mu \mathrm{g} / \mathrm{L}$ in water, 2-methylisoborneol at $0.002-0.1 \mu \mathrm{g} / \mathrm{L}$ in water, and $\alpha$-terpineol at $4.6 \mu \mathrm{g} / \mathrm{L}$ in water), as well as notable "green" and "musty" odors. It is also worth noting that they reported the odor thresholds of these identified terpenoid compounds in apple juice were perceived at lower concentrations than previously published odor thresholds in water (Siegmund and Pöllinger-Zierler, 2006).

The average concentrations of the terpenoid compounds in the objectionable milk samples after $\mathrm{d} 0$ and 14 are shown in Figure 2; again, these compounds were not detected in the control milk. Over the 14-d storage period, endo-borneol and $\alpha$-terpineol decreased significantly $(P<0.05)$. Endo-borneol decreased approximately 5 -fold from 0.95 to $0.20 \mu \mathrm{g} / \mathrm{L}$ and $\alpha$-terpineol decreased about $26 \%$, from 0.84 to $0.62 \mu \mathrm{g} / \mathrm{L}$, whereas 2-methylisoborneol increased significantly $(P<0.05)$ from 0.0037 to $0.017 \mu \mathrm{g} / \mathrm{L}$, approximately a 4-fold increase. The concentration of 2-methylisoborneol was well above its published odor threshold of $0.002 \mu \mathrm{g} / \mathrm{L}$ in water. The survival of microbial strains in pasteurized milk may have resulted in the noted increase of 2-methylisoborneol during storage (Siegmund and Pöllinger-Zierler, 2006). The decrease in concentration of endo-borneol and $\alpha$-terpineol indicated that these products were not stable over time in the milk samples. It is uncertain whether the loss of endo-borneol and $\alpha$-terpineol in the milk samples was directly related to the increase of 2-methylisoborneol over time, either through microbial metabolism or other conversion pathways. Further study would be necessary to elucidate these mechanisms.

As a first step to screen the effect of the noted changes in lipid oxidation and terpenoid compounds on the observed objectionable flavor attributes of the SOF-labeled milk, a preliminary sensory recombination study was conducted with 3 experienced dairy judges. Nonflavor defective milk (control) was dosed with either the lipid oxidation products (2-heptanone, 2-nonanone, E-2-decenal, E,E-2,4-decadienal, nonanal) or the terpenoid compounds (endo-borneol, 2-methylisoborneol, $\alpha$-terpineol), or a combination of all compounds, at equivalent concentrations of the $\mathrm{d} 0$ and 
14 SOF-labeled samples. The recombination sample with the lipid oxidation compounds was not found to be objectionable by any of the judges, and thus the compounds were excluded from further sensory evaluation. However, evaluation of the recombination milk sample with the terpenoid compounds were perceived as unclean with distinctly unpleasant aftertastes and matched the initial sensory screening of the purported SOF milk samples at the time of reception from the dairy farm. Finally, the recombination sample containing all selected volatile compounds was described in a similar manner as the recombination sample with only terpenoid compounds. Therefore, the terpenoid compounds were selected for further sensory evaluation.

A degree of difference test was employed to determine the magnitude of sensory change and assess the relevance of the identified terpenoid compounds. Three samples were evaluated, a blind control milk (same as reference) and milk samples spiked with the terpenoid compounds at concentrations determined at $\mathrm{d} 0$ and at d 14, along with a reference milk; the results are shown in Table 6. In comparison to the blind control reference milk, the milk spiked with compounds at equivalent concentrations to d 0 SOF milk samples showed an increased difference score (mean score $=1.7, P$-value
$=0.06)$, whereas the milk spiked with d 14 levels was highly statistically different (mean score $=2.0, P$-value $<0.01$ ). The panelists described the spiked milk samples as "stale," "pine-like," "dirty," "cardboard," "old," "unclean," "musty," "earthy," "grassy," and "metallic." Descriptors such as stale, cardboard, and grassy have historically been used to describe purported SOF milk samples as well (Shipe et al., 1978; Nicholson, 1993; Juhlin et al., 2010b); however, in the current study the source of these objectionable flavors was found to be of microbial origin, and not lipid oxidation.

Microbial flavor taints can arise from microorganisms of aqueous or terrestrial sources within the milk supply chain, for example through environmental contact or contamination, feed or water sources, silage, the milking process, commingled milk at the processing site, and so on (Siegmund and Pöllinger-Zierler, 2006). The SOF milk has frequently been associated with seasonal occurrences, which points to a variability in animal conditions or husbandry practices. A seasonal alteration could likewise result in microbial contamination, growth, and subsequent negative flavor development in milk. Additionally, thermoacidophilic bacteria capable of surviving pasteurization could result in changes in milk quality during subsequent storage. This would ac-

Table 5. Aroma compounds identified in the neutral/basic fraction of spontaneous oxidized flavor labeled milk ${ }^{1}$

\begin{tabular}{|c|c|c|c|c|c|}
\hline \multirow[b]{2}{*}{ Identified compound } & \multirow[b]{2}{*}{ DB-WAX ${ }^{2}$} & \multirow[b]{2}{*}{$\mathrm{HP}-5^{2}$} & \multirow[b]{2}{*}{ Odor descriptor } & \multicolumn{2}{|c|}{ Average OSME intensity } \\
\hline & & & & d 0 & d 14 \\
\hline Propanoic acid & 1523 & 687 & Pungent, rancid & - & 1.8 \\
\hline Pentanal & - & 732 & Pungent & 2.2 & 2.0 \\
\hline Hexanal & 1107 & 777 & Oxidized, fat & 1.7 & 1.0 \\
\hline Butyric acid & - & 816 & Cabbage, onion & - & 1.7 \\
\hline Hexanol & 1347 & 844 & Hay, grass & 1.7 & 1.8 \\
\hline 2-Heptanone & 1195 & 882 & Fatty & 1.7 & 2.3 \\
\hline 3-Octanol & 1347 & 981 & Mushroom, earthy & 2.0 & 2.0 \\
\hline Benzyl alcohol & 1872 & 1040 & Roasted, meaty & 2.2 & 1.8 \\
\hline 1-Octanol & 1550 & 1076 & Chemical, metal & 2.3 & 1.7 \\
\hline 2-Nonanone & 1435 & 1090 & Green & 2.3 & 2.0 \\
\hline Nonanal & 1347 & 1112 & Fat, cream & 2.0 & 2.3 \\
\hline Endo-borneol & 1657 & 1169 & Paper, musty & 2.2 & 2.7 \\
\hline 2-Methylisoborneol & 1573 & 1175 & Musty & 2.3 & 3.7 \\
\hline Octanoic acid & - & 1179 & Sweat, cheese & 2.3 & 2.5 \\
\hline Naphthalene & 1696 & 1189 & Musty & - & 2.5 \\
\hline$\alpha$-Terpineol & 1678 & 1192 & Musty & 2.3 & 2.3 \\
\hline Decanal & 1550 & 1197 & Green, fat & 2.3 & 2.2 \\
\hline E-2-Decenal & 1573 & 1240 & Cheese, fat & 1.8 & 2.0 \\
\hline E,E-2,4-decadienal & 1642 & 1252 & Paper, cardboard, bean & 2.0 & 2.0 \\
\hline$\delta$-Octalactone & - & 1270 & Peach, coconut & 2.2 & 2.3 \\
\hline Decanol & 1696 & 1272 & Fat & 2.0 & 2.7 \\
\hline$\delta$-Nonalactone & 2163 & 1281 & Coconut & 2.3 & 2.7 \\
\hline p-Vinyl guaiacol & 2280 & 1344 & Malty, mushroom & 2.2 & 2.8 \\
\hline$\gamma$-Nonalactone & - & 1366 & Coconut, peach & 1.8 & 2.3 \\
\hline Decanoic acid & - & 1373 & Rancid, fat & 2.5 & 2.2 \\
\hline$\delta$-Decalactone & - & 1500 & Lactone, cream & 2.5 & 2.7 \\
\hline Dodecanoic acid & - & 2169 & Oil, soap & - & 1.8 \\
\hline
\end{tabular}

${ }^{1}$ Identified on 2 column chemistries with corresponding odor descriptors and OSME odor intensity values from d 0 and 14 post-HTST pasteurization, averaged among 3 panelists.

${ }^{2}$ DB-WAX and HP-5 columns were from Agilent Technologies, Santa Clara, California. 


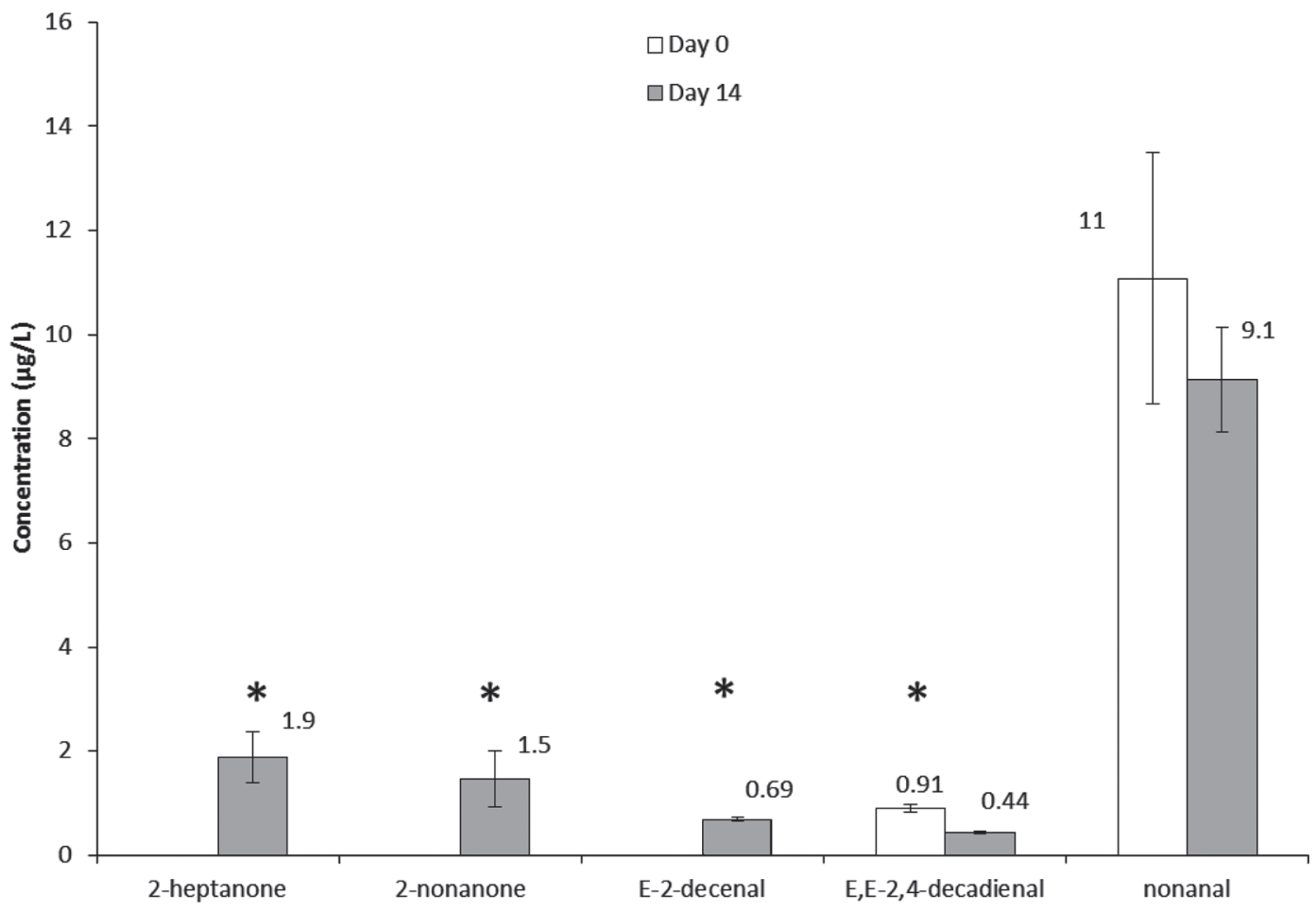

Figure 1. Concentrations of lipid oxidation compounds in off-flavored spontaneous oxidized flavor labeled milk, $\mathrm{d} 0$ and 14 . Average concentrations are reflected in triplicate with respective SD. Average concentrations marked with an asterisk $\left(^{*}\right)$ were significantly different between d 0 and $14(P<0.05)$.

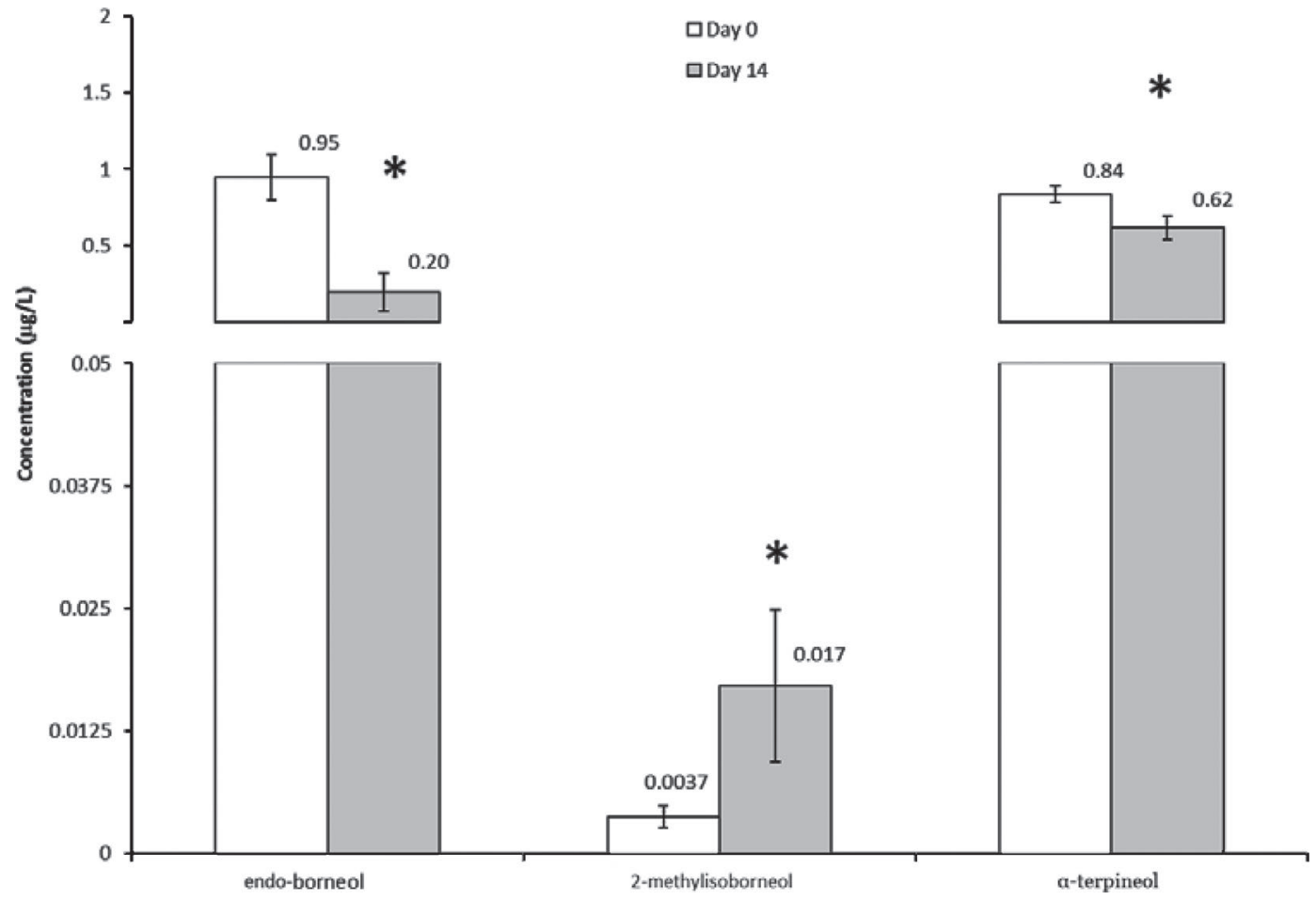

Figure 2. Concentrations of terpenoid compounds present in off-flavored spontaneous oxidized flavor labeled milk, d 0 and 14 . Average concentrations are reflected in triplicate with respective SD. Average concentrations marked with an asterisk $(*)$ were significantly different between $\mathrm{d} 0$ and $14(P<0.05)$. 
Table 6. Sensory degree of difference of a reference milk, a blind control milk, and milk spiked with 2 levels of terpenoid compounds reported in milk at d 0 and 14

\begin{tabular}{lcc}
\hline Milk sample & Mean rating & $P$-value \\
\hline Blind control & 0.7 & - \\
Recombinant d 0 & 1.7 & 0.06 \\
Recombinant d 14 & 2.0 & $<0.01$
\end{tabular}

${ }^{1}$ Panelists evaluated samples using a 5 -point scale: $0=$ no difference, 1 $=$ very slight difference, $2=$ slight difference, $3=$ moderate difference, $4=$ large difference, or $5=$ extremely large difference; $P$-value using a 2-sided Dunnett's multiple comparisons test.

count for changes in the flavor taint profile over time, as reported in Figure 2.

\section{CONCLUSIONS}

After further investigation of the objectionable flavored milk flagged as SOF, the source was reported to arise from microbial flavor taints endo-borneol, 2-methylisoborneol, and $\alpha$-terpineol. Lipid oxidation compounds, although identified in both the control and SOF labeled milk samples in this study, were typically of high quality milk and not shown to reproduce the objectionable flavor profile. Therefore, the SOF milk was inaccurately labeled, indicating the challenges of classifying and investigating related flavor problems without adequate identification of the active compounds. More work is needed to understand the prevalence of microbial taints in the milk supply, the effect of thermal processing on microbial survival, and the source of the microbial strains involved. Furthermore, characterizing the flavor profiles from other SOF-labeled milk could potentially reveal other sources for the noted flavor defects. Identification of the sources of objectionable flavors in milk provides critical control points in dairy production and distribution to maintain high product quality.

\section{ACKNOWLEDGMENTS}

The authors of this study thank the Midwest Dairy Association (St. Paul, MN) and the Agriculture Utilization Research Institute (Crookston, MN) for provided support and resources for completion of this project.

\section{REFERENCES}

Bruhn, J. C., A. A. Frank, and G. S. Goble. 1976. Factors relating to development of spontaneous oxidized flavor in raw milk. J. Dairy Sci. 59:828-833.
Buttery, R. G., J. G. Turnbaugh, and L. C. Ling. 1988. Contribution of volatiles to rice aroma. J. Agric. Food Chem. 36:1006-1009.

Clausen, M. R., C. Connolly, L. H. Skibsted, and J. Stagsted. 2010. Oxidative stability of bovine milk determined by individual variability in herd irrespective of selenium status. Int. Dairy J. 20:507513.

Colahan-Sederstrom, P. M., and D. G. Peterson. 2005. Inhibition of key aroma compound generated during ultrahigh-temperature processing of bovine milk via epicatechin addition. J. Agric. Food Chem. 53:398-402.

Dunkley, W. L., M. Ronning, and J. Voth. 1963. Influence of injection of cows with copper glycinate on blood and milk copper and oxidized flavor in milk. J. Dairy Sci. 46:1059-1063.

Engel, W., W. Bahr, and P. Schieberle. 1999. Solvent assisted flavour evaporation-a new and versatile technique for the careful and direct isolation of aroma compounds from complex food matrices. Eur. Food Res. Technol. 209:237-241.

Granelli, K., P. Barrefors, L. Björck, and L.-A. Appelqvist. 1998. Further studies on lipid composition of bovine milk in relation to spontaneous oxidised flavour. J. Sci. Food Agric. 77:161-171.

Juhlin, J., W. F. Fikse, I.-L. Orde-Ostrom, P. Barrefors, and A. Lunden. 2010a. Factors relating to incidence of spontaneous oxidized flavor and copper in cow's milk. Acta Agric. Scand. 60:94-103.

Juhlin, J., F. Fiske, A. Lundén, J. Pckova, and S. Agenäs. 2010b. Relative impact of $\alpha$-tocopherol, copper and fatty acid composition on the occurrence of oxidized milk flavour. J. Dairy Res. 77:302-309.

Karagül-Yüceer,, Y., M. Drake, and K. Cadwallader. 2001. Aromaactive components of nonfat dry milk. J. Agric. Food Chem. 49:2948-2953.

McDaniel, M. R., R. Miranda-Lopez, B. T. Watson, N. J. Micheals, and L. M. Libbey. 1990. Pinot noir aroma: A sensory/gas chromatographic approach. Page 23 in Flavors Off-Flavors '89. G. Charalambous, ed. Elsevier, Amsterdam, the Netherlands.

Nicholson, J. W. G. 1993. Spontaneous Oxidized Flavour in Cow's Milk. International Dairy Federation, Schaerbeek, Belgium.

Plotto, A., C. A. Margaría, K. L. Goodner, R. Goodrich, and E. A. Baldwin. 2004. Odour and flavour thresholds for key aroma components in an orange juice matrix: Terpenes and aldehydes. Flavour Fragr. J. 19:491-498. https://doi.org/10.1002/ffj.1470.

Schrader, K. K., and S. T. Summerfelt. 2010. Distribution of off-flavor compounds and isolation of geosmin-producing bacteria in a series of water recirculation systems for rainbow trout culture. N. Am. J. Aquaculture 72:1-9.

Shipe, W. F., R. Bassette, D. D. Deane, W. L. Dunkley, E. G. Hammond, W. J. Harper, D. H. Kleyn, M. E. Morgan, J. H. Nelson, and R. A. Scanlan. 1978. Off flavors of milk: Nomenclature, standards, and bibliography. J. Dairy Sci. 61:855-869. https://doi.org/ 10.3168/jds.S0022-0302(78)83662-5.

Siegmund, B., and B. Pöllinger-Zierler. 2006. Odor thresholds of microbially induced off-flavor compounds. J. Agric. Food Chem. 54:5984-5989.

Teranishi, R., R. G. Buttery, and D. G. Gaudagni. 1974. Odor quality and chemical structure in fruit and vegetable flavors. Ann. N. Y. Acad. Sci. 237:209-216.

Timmons, J. S., W. P. Weiss, D. L. Palmquist, and W. J. Harper. 2001. Relationships among dietary roasted soybeans, milk components, and spontaneous oxidized flavor of milk. J. Dairy Sci. 84:2440-2449.

Villeneuve, M.-P., Y. Lebeuf, R. Gervais, G. F. Tremblay, J. C. Vuillemard, J. Fortin, and P. Y. Chouinard. 2013. Milk volatile organic compounds and fatty acid profile in cows fed timothy as hay, pasture, or silage. J. Dairy Sci. 96:7181-7194. https://doi.org/10 $.3168 /$ jds.2013-6785. 